\title{
Prehospital score for acute disease: a community-based observational study in Japan
} Yasuhiro Toyoda $^{\dagger 1}$, Yoshio Matsuo
Hidekazu Fujiwara $^{\dagger 4}$, Toshio Takatorige

\author{
Address: ${ }^{1}$ Public Health, Department of Social and Environmental Medicine, Graduate School of Medicine, Osaka University, Osaka, Japan, \\ ${ }^{2}$ Emergency Division, Kishiwada Tokushukai Hospital, Kishiwada, Japan, ${ }^{3}$ Emergency Division, Kishiwada City Hospital, Kishiwada, Japan and \\ ${ }^{4}$ Kishiwada City Fire Department, Kishiwada, Japan \\ Email: Yasuhiro Toyoda - ytoyoda@pbhel.med.osaka-u.ac.jp; Yoshio Matsuo - kch-25_q9q9@kch.city.kishiwada.osaka.jp; \\ Hiroyuki Tanaka - ertanaka@tokyo-med.ac.jp; Hidekazu Fujiwara - kishiwada-kyukyu@tvk.zaq.ne.jp; \\ Toshio Takatorige - takatorige@pnhel.med.osaka-u.ac.jp; Hiroyasu Iso* - iso@pbhel.med.osaka-u.ac.jp \\ * Corresponding author †Equal contributors
}

Published: 15 October 2007

BMC Emergency Medicine 2007, 7:17 doi:10.1186/147I-227X-7-17
Received: 18 May 2007

Accepted: 15 October 2007

This article is available from: http://www.biomedcentral.com/I47/-227X/7//7

(c) 2007 Toyoda et al.; licensee BioMed Central Ltd.

This is an Open Access article distributed under the terms of the Creative Commons Attribution License (http://creativecommons.org/licenses/by/2.0), which permits unrestricted use, distribution, and reproduction in any medium, provided the original work is properly cited.

\begin{abstract}
Background: Ambulance usage in Japan has increased consistently because it is free under the national health insurance system. The introduction of refusal for ambulance transfer is being debated nationally. The purpose of the present study was to investigate the relationship between prehospital data and hospitalization outcome for acute disease patients, and to develop a simple prehospital evaluation tool using prehospital data for Japan's emergency medical service system.
\end{abstract}

Methods: The subjects were 9,160 consecutive acute disease patients aged $\geq 15$ years who were transferred to hospital by Kishiwada City Fire Department ambulance between July 2004 and March 2006. The relationship between prehospital data (age, systolic blood pressure, pulse rate, respiration rate, level of consciousness, $\mathrm{SpO}_{2}$ level and ability to walk) and outcome (hospitalization or non-hospitalization) was analyzed using logistic regression models. The prehospital score component of each item of prehospital data was determined by beta coefficients. Eligible patients were scored retrospectively and the distribution of outcome was examined. For patients transported to the two main hospitals, outcome after hospitalization was also confirmed.

Results: A total of 8,330 (91\%) patients were retrospectively evaluated using a prehospital score with a maximum value of $\mathrm{I} 4$. The percentage of patients requiring hospitalization rose from $9 \%$ with score $=0$ to $100 \%$ with score $=14$. With a cut-off point score $\geq 2$, the sensitivity, specificity, positive predictive value and negative predictive value were $97 \%, 16 \%, 39 \%$ and $89 \%$, respectively. Among the 6,498 patients transported to the two main hospitals, there were no deaths at scores $\leq \mathrm{I}$ and the proportion of non-hospitalization was over $90 \%$. The proportion of deaths increased rapidly at scores $\geq \mathrm{II}$.

Conclusion: The prehospital score could be a useful tool for deciding the refusal of ambulance transfer in Japan's emergency medical service system. 


\section{Background}

The Japanese national emergency medical service system has been established since 1963 and enables those in need of urgent medical treatment to summon an ambulance by calling the free national emergency telephone number '119'. Approximately $78 \%$ of ambulances are staffed by emergency life-saving technicians, who were introduced in 1991. Prior to that, emergency medical technicians played a major role in prehospital settings but were only allowed to perform basic life support procedures such as external chest compression and ventilation with a bag valve mask. Emergency life-saving technicians, by contrast, are permitted to perform endotracheal intubation, defibrillation, intravenous infusion of Ringer's solution, and administration of epinephrine. However, these treatments are allowed only for cardiopulmonary arrest patients. In addition, they are not permitted to carry out additional life-saving interventions and clinical tests such as needle thoracostomy, cricothyroidotomy, 12-lead electrocardiograms, blood glucose measurements and administration of drugs other than epinephrine. Furthermore, the frequency with which vital signs are taken at the scene is still low in Japan; 25\% for blood pressure and $27 \%$ for $\mathrm{SpO}_{2}$ [1]. As a result, prehospital care in Japan is very limited compared with to in western countries [2-5].

Meanwhile, the Japanese national medical insurance system and free ambulance call-outs have resulted in a lack of concern over ambulance usage. This usage has increased consistently by over 5 million cases per year since 2004 . According to national data, 51\% of these were mild cases that did not require hospitalization [1]. In addition, the rapid aging of society, with more than $19 \%$ of the population aged over 65 years in 2004 [6], has promoted an increase in ambulance usage, especially for acute disease $[7,8]$.

In this situation, refusal of ambulance transfer for mild cases is being debated nationally. The fire and disaster management agency established a 'Committee for demand of ambulance usage' in 2006. For appropriate ambulance usage for hospitalization, a simple triage tool to decide the refusal of ambulance usage would be useful.

The purpose of this study was to investigate the relationship between prehospital data and hospitalization outcome for acute disease patients aged $\geq 15$ years, and to develop a simple tool for deciding the refusal of ambulance usage based primarily on vital signs.

\section{Methods \\ Study setting}

This study was conducted in Kishiwada City, Osaka Prefecture, Japan, which has a population of about 200,000 and an area of $72 \mathrm{~km}^{2}$. Kishiwada City Fire Department controls the emergency medical service system. A total of 17,293 patients were transferred by the Kishiwada City Fire Department ambulances between July 2004 and March 2006. The subjects in the present study were 9,160 consecutive acute disease patients aged $\geq 15$ years during this period.

All ambulances in Kishiwada carry at least one emergency life-saving technician who records prehospital data such as patient age, vital signs, chief complaint and simple physical examinations, taken at the scene or in the ambulance. Since April 2004, prehospital data have been entered into the computer database by the captain of each ambulance, who has the emergency life-saving technician national license. Approximately $80 \%$ of emergency patients are transferred to the two main hospitals; Kishiwada City Hospital and Kishiwada Tokushukai Hospital. Five or more clinical physicians, four or more emergency nurses, one or more radiology technicians, one or more clinical laboratory technicians and one or more pharmacists reside in these hospitals over each 24-hour period. These hospitals are certificated as emergency hospitals by the Japan Acute Medicine Association.

\section{Study design and protocol}

We investigated the relationship between prehospital data and hospitalization outcome for the acute disease patients transported by the Kishiwada City Fire Department ambulances, using a multivariable logistic regression model, and developed the prehospital score by the beta coefficients of significant variables.

The following prehospital data collected by emergency personnel, including emergency life-saving technicians, were extracted from the database: age, systolic blood pressure, pulse rate, consciousness level, $\mathrm{SpO}_{2}$ and ability to walk. These are routinely taken at the scene or in the ambulance and are relatively objective.

The outcome was reported as hospitalization or non-hospitalization. The chief of the emergency section of Kishiwada City Fire Department, a trained emergency lifesaving technician, confirmed the hospitalization outcome and the final diagnosis by the attending physicians using FAX or telephone. Deaths in the emergency room were regarded as hospitalization. In addition, the outcome after hospitalization was confirmed for patients transferred to the two main hospitals (Kishiwada City Hospital and Kishiwada Tokushukai Hospital), i.e. $71 \%$ of the total number of subjects.

The continuous variables - age, systolic blood pressure, pulse rate and $\mathrm{SpO}_{2}$ - were converted to categorical variables for practical use. We decided the reference of each continuous variable according to normal clinical range. 
Systolic blood pressure levels were categorized as $<80,90-$ 99, 100-149 (reference), 150-159, 160-169, 170-179, 180-189, 190-199, and $\geq 200 \mathrm{mmHg}$; pulse rates were categorized as <50, 50-59, 60-89 (reference), 90-99, 100-109, 110-119, and $\geq 120$ beats per min; $\mathrm{SpO}_{2}$ was categorized into <95\% (hypoxic) and $\geq 95 \%$ (normal). Patients with capillary circulation failure whose $\mathrm{SpO}_{2}$ levels could not be measured were also regarded as hypoxic. Ages were categorized as 15-59, 60-69, 70-79, 80-89 and $\geq 90$ years; the consciousness level was categorized according to the Japan Coma Scale (JCS) as 0, I, II and III; the ability to walk was categorized as "yes" or "no". Cases in which the emergency life-saving technician advised the patient not to walk were regarded as "no". The major category of the Japan Coma Scale (JCS), a widely used criterion for evaluation of consciousness in Japan, was used in the present study. The scale is $0=$ alert, $\mathrm{I}=$ awake without stimulation, II = awake with stimulation, III = not awake with stimulation [9]. When multiple data were available for each patient, the initial data were used for analyses.

The prehospital score was determined as a simple integer $(0,1,2$ or 3$)$ for practical use on the basis of the beta coefficient for each independent variable. The total score was obtained from the sum of all items of prehospital data.

Eligible patients were retrospectively scored and the distributions of all scores for the hospitalized and non-hospitalized groups were examined. The proportion hospitalized was calculated and related to the total score. Moreover, for patients transported to the two main hospitals (Kishiwada City Hospital and Kishiwada Tokushukai Hospital), the proportions (1) non-hospitalized, (2) discharged after hospitalization, (3) transported to another hospital and (4) died in the emergency room or after hospitalization were related to the total score.

The sensitivity, specificity, positive predictive value (PPV) and negative predictive value (NPV) for predicting hospitalization were calculated at each score point, and the area under the receiver operating characteristic (ROC) curve was determined with 95\% confidence intervals.

The statistical analysis package SPSS 12.0J for Windows (SPSS Japan inc., Tokyo, Japan) was used for data analyses. All p-values were two-tailed, and p-values $<0.05$ were considered statistically significant.

\section{Ethical approval}

Prehospital and outcome data did not include personal information. The present study was approved by the ethical committee of the Graduate School of Medicine, Osaka University, Japan.

\section{Results \\ Multivariable logistic regression and development of prehospital score}

From a total of 9,169 patients, complete data were available for 8,330 (91\%), which were used for the analyses. Of these, $36 \%(3,002)$ were hospitalized. The mean age $( \pm$ $\mathrm{SD})$ of the hospitalization group was $70 \pm 16$ years while that of the non-hospitalization group was $58 \pm 20$ years.

Table 1 shows the beta coefficient and multivariable odds ratios for the components of prehospital score. All independent variables - age, systolic blood pressure, pulse rate, level of consciousness, $\mathrm{SpO}_{2}$ and ability to walk were statistically significant in predicting hospital outcome.

The prehospital score component was equated to 1, 2 or 3 when the beta coefficient was $<0.5,0.5-1.0$ or $\geq 1.0$, respectively. The total score calculated by adding the six component scores to give a value from 0 to 14 .

\section{Results of retrospective scoring}

The distribution of total scores for the hospitalization and non-hospitalization groups is shown in Figure 1; the hospitalization group follows an approximately normal distribution, while the non-hospitalization group is skewed with most patients receiving a low score. The modal score of the hospitalization group was 4 while that of the nonhospitalization group was 2 .

Figure 2 shows the proportion and 95\% confidence interval of hospitalization in relation to total score. A linear relationship was observed between the score and the proportion hospitalized: $9 \%$ of patients with a score of 0 required hospitalization, and this increased to $100 \%$ for those with a score of 13 .

Among the 102 patients requiring hospitalization with a total score $\leq 1,55$ (51\%) were diagnosed with digestive disease, including 10 with gastrointestinal bleeding, 5 with cholangitis, 6 with pancreatitis and 7 with appendicitis. This contrasts with the overall proportion of digestive disease of $19 \%$. In addition, 16 patients (15\%) were diagnosed with psychiatric disease and 12 (7\%) with cerebral disease. This compares with overall proportions of $7 \%$ for psychiatric disease and 19\% for cerebral disease. Only 14 patients were not hospitalized despite a high total score $(\geq 11)$. These included 4 patients suffering from a hypoglycemic attack, 7 with loss of consciousness and suspected vasovagal syncope, 2 with atrial arrhythmia and 1 with malignancy. Among the 165 patients who died in the emergency room, one had a total score of 4 , three scored 7 , one scored 8 , seven scored 9 and the remainder scored $\geq 10$. Those patients scoring 4-9 rapidly underwent 
Table I: Multivariable odds ratios of hospitalization in relation to prehospital score

\begin{tabular}{|c|c|c|c|c|}
\hline Prehospital data & No. of hospitalization/patients & beta coefficient & OR $(95 \% \mathrm{Cl})$ & Prehospital Score \\
\hline \multicolumn{5}{|l|}{ Age, $y$} \\
\hline$<60$ & $639 / 3,101$ & 0 & I & 0 \\
\hline $60-69$ & $513 / 1,465$ & 0.53 & $1.69(1.45-1.97)^{*}$ & 2 \\
\hline 70-79 & $939 / 2,098$ & 0.83 & $2.30(2.01-2.64)^{*}$ & 2 \\
\hline $80-89$ & $730 / 1,363$ & 1.04 & $2.82(2.42-3.29)^{*}$ & 3 \\
\hline$>=90$ & $181 / 303$ & 1.15 & $3.16(2.41-4.13)^{*}$ & 3 \\
\hline \multicolumn{5}{|c|}{ Systolic blood pressure, $\mathrm{mmHg}$} \\
\hline$<80$ & $239 / 277$ & 0.93 & $2.53(1.63-3.94)^{*}$ & 2 \\
\hline $80-89$ & $96 / 185$ & 0.55 & $1.73(1.25-2.41)^{*}$ & 2 \\
\hline $90-99$ & $158 / 428$ & -0.05 & $0.95(0.75-1.20)$ & 0 \\
\hline $100-149$ & $1,489 / 4,512$ & 0 & I & 0 \\
\hline $150-159$ & $236 / 767$ & -0.14 & $0.87(0.73-1.05)$ & 0 \\
\hline $160-169$ & $223 / 659$ & -0.04 & $0.97(0.80-1.23)$ & 0 \\
\hline $170-179$ & $134 / 479$ & -0.01 & $0.99(0.80-1.23)$ & 0 \\
\hline $180-189$ & $134 / 397$ & -0.09 & $0.91(0.72-1.16)$ & 0 \\
\hline $190-199$ & $81 / 215$ & 0.14 & $1.15(0.84-1.56)$ & 0 \\
\hline$>=200$ & $|82 / 4| \mid$ & 0.37 & $1.45(1.15-1.81)^{*}$ & I \\
\hline \multicolumn{5}{|l|}{ Pulse, per min } \\
\hline$<50$ & $208 / 235$ & 0.92 & $2.5 \mathrm{I}(1.52-4.13)^{*}$ & 2 \\
\hline $50-59$ & $90 / 273$ & -0.03 & $0.97(0.73-1.29)$ & 0 \\
\hline $60-89$ & $1,265 / 4,238$ & 0 & 1 & 0 \\
\hline $90-99$ & $447 / 1,372$ & 0.09 & $1.10(0.95-1.27)$ & 0 \\
\hline $100-109$ & $366 / 954$ & 0.23 & $1.26(1.07-1.49)^{*}$ & 1 \\
\hline $110-119$ & $245 / 54 I$ & 0.52 & $1.67(1.37-2.05)^{*}$ & 2 \\
\hline $120-129$ & $218 / 437$ & 0.63 & $1.88(1.50-2.35)^{*}$ & 2 \\
\hline$>=130$ & $163 / 280$ & $0.7 \mathrm{I}$ & $2.02(1.53-2.67)^{*}$ & 2 \\
\hline \multicolumn{5}{|c|}{ Consciousness, JCS } \\
\hline 0 & $1,990 / 6,699$ & 0 & I & 0 \\
\hline 1 & $485 / 942$ & 0.51 & $1.67(1.44-1.94)^{*}$ & 2 \\
\hline II & $188 / 283$ & 1.09 & $2.98(2.27-3.90)^{*}$ & 3 \\
\hline III & $339 / 406$ & 1.35 & $3.84(2.82-5.23)^{*}$ & 3 \\
\hline \multicolumn{5}{|l|}{ Saturation $\mathrm{O}_{2}, \%$} \\
\hline$>=95$ & $\mathrm{I}, 804 / 6,57 \mid$ & 0 & 1 & 0 \\
\hline$<95$ & I, I 98/I,759 & 0.99 & $2.69(2.36-3.06)^{*}$ & 2 \\
\hline \multicolumn{5}{|l|}{ Ability to walk } \\
\hline yes & $389 / 2,228$ & 0 & I & 0 \\
\hline no & $2,613 / 6,102$ & 0.73 & $2.08(1.82-2.37)^{*}$ & 2 \\
\hline
\end{tabular}

$\mathrm{Cl}$; Confidence Interval JCS; Japan coma scale OR; Odds ratio

$\mathrm{P}$ value for logistic regression analysis ; $* \mathrm{P}<0.01$

cardiopulmonary arrest following headache, chest pain, dyspnea, fatigue and loss of consciousness.

Figure 3 shows the proportions of patients transported to the two main hospitals who were non-hospitalized, discharged after hospitalization, transferred to another hospital or died ( $n=6,498,71 \%$ of the subjects). The proportion non-hospitalized declined, and the proportions discharged after hospitalization, transferred to another hospital or died all increased linearly, between scores 2 and 10 . At score 0 or 1, there were no deaths and the proportion non-hospitalized was over $90 \%$. The proportion discharged after hospitalization was higher than the proportion who died at scores $\leq 10$. The proportion who died increased rapidly at scores $\geq 11$ and exceeded $80 \%$ at scores $\geq 12$. Among these 6,498 patients, 14 with total scores $\leq 3$ died after hospitalization, including 7 with malignancy, 3 with liver cirrhosis and 2 with cardiovascular disease. 


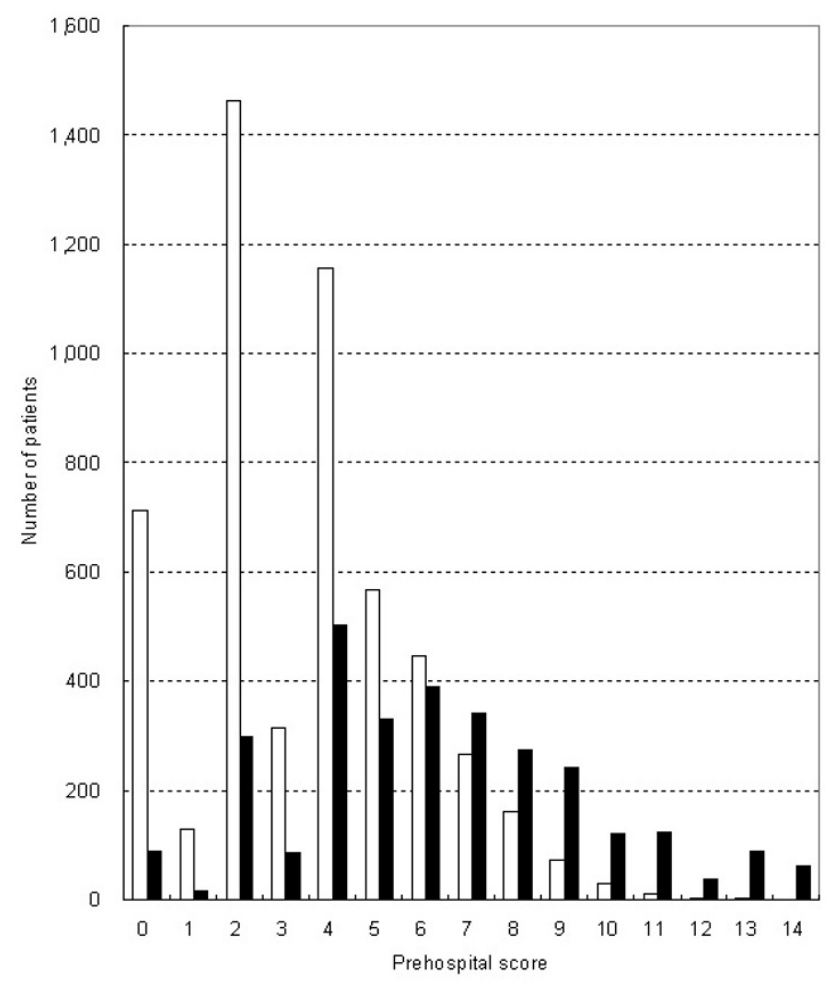

$\square$ Non-hospitalization $\mathbf{a}$ Hospitalization

Figure I

The number of patients according to prehospital

score. The modal score of the hospitalization group was 4 while that of the non-hospitalization group was $2(n=8,330)$.

\section{Sensitivity and specificity of prehospital score}

Figure 4 shows the ROC curve; the area under the curve is 0.75 (95\% confidence interval 0.74-0.76, $\mathrm{n}=8,330$ ). When that was set at $\geq 2$, the sensitivity, specificity, PPV and NPV were 97\%, 16\%, 39\% and $89 \%$, respectively (Table 2).

\section{Discussion}

This study is an investigation of the relationship between prehospital data and requirement for hospitalization in acute disease patients aged $\geq 15$ years; it also details the development of a simple triage assessment tool. Age, systolic blood pressure, pulse rate, level of consciousness, $\mathrm{SpO}_{2}$ level and ability to walk were found to be statistically significant predictors for hospitalization requirement. A prehospital score ranging from 0 to 14 was developed on the basis of the multivariable analysis results.

In western countries, prehospital triage guidelines are based on patient symptoms for acute disease [10-16], but these are inappropriate for use in Japan as emergency lifesaving technicians can only perform limited medical treatments.

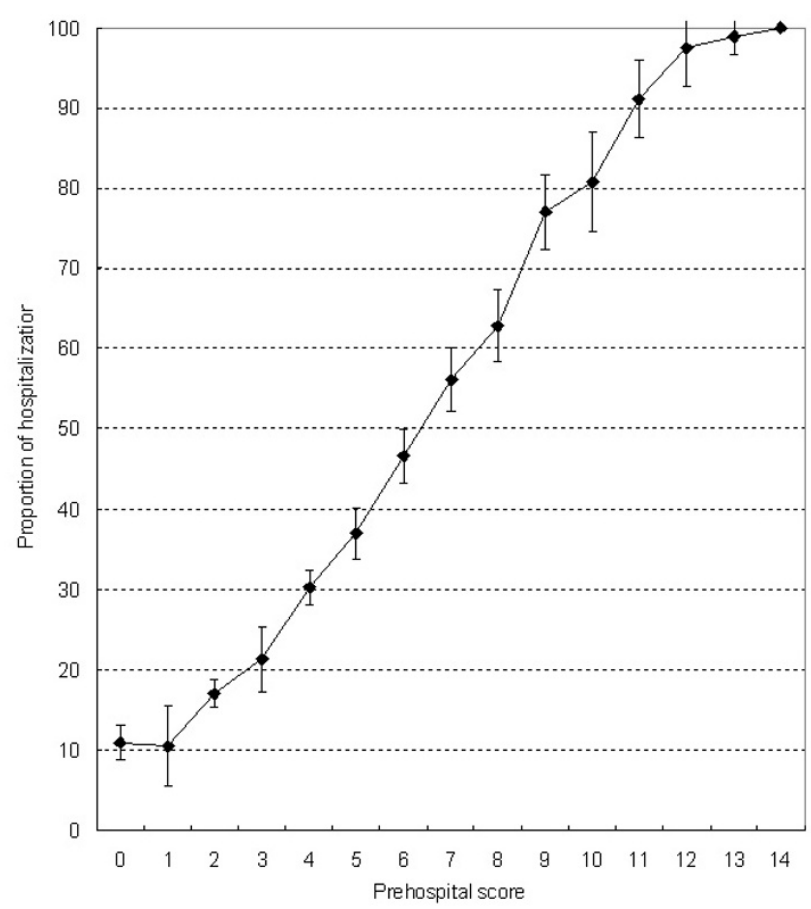

Figure 2

The proportion hospitalized (with $95 \%$ confidence intervals) in relation to prehospital score. A linear relationship was observed between the score and the proportion hospitalized $(n=8,330)$.

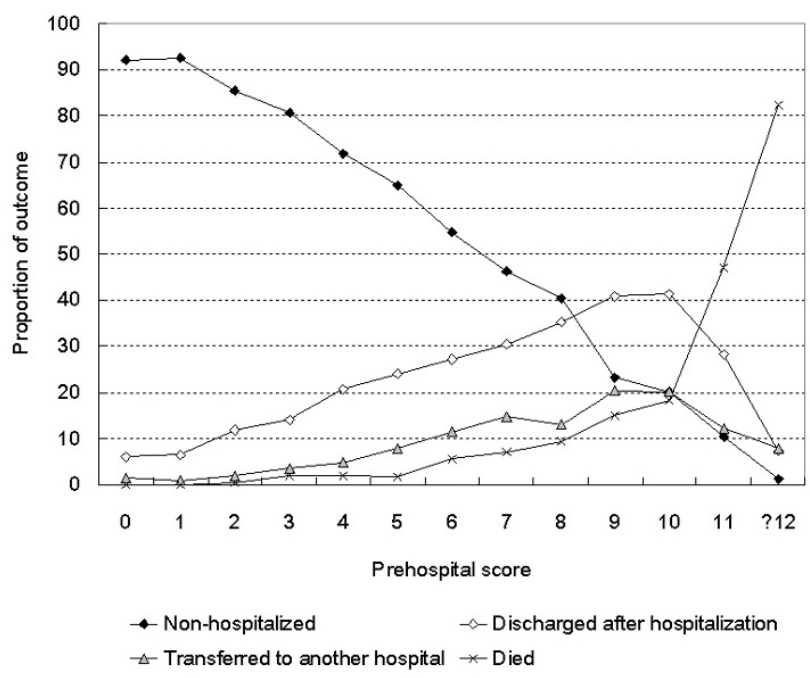

Figure 3

Outcome proportions in relation to prehospital score. The outcome proportions including after hospitalization were calculated for patients transported to the two main hospitals. The proportion discharged after hospitalization was higher than the proportion who died at scores $\leq 10$, and vice versa at scores $\geq I I(n=6,498)$. 


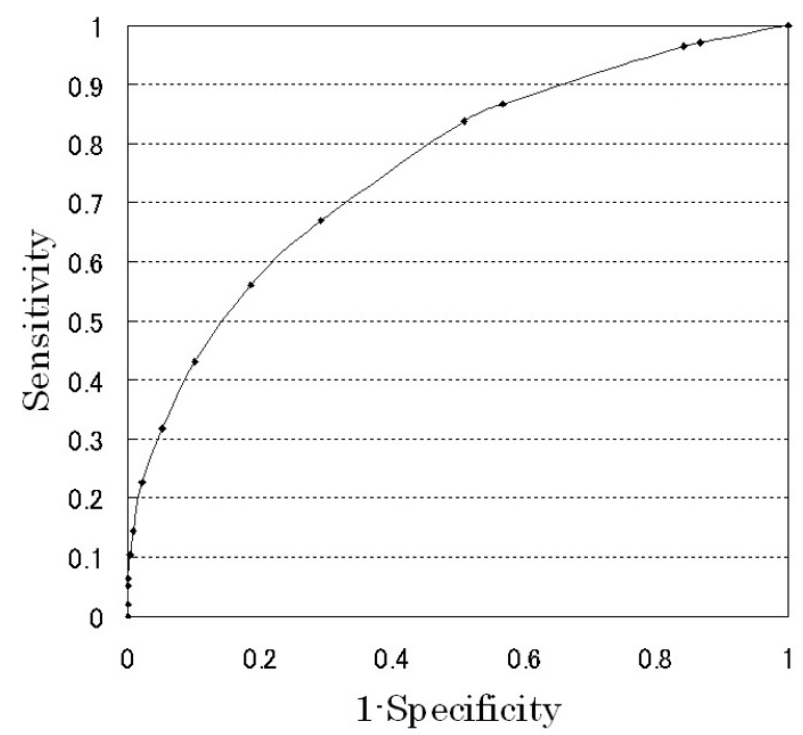

Figure 4

ROC curve for prehospital score to predict hospitalization. The area under the curve was 0.75 (95\% confidence intervals $0.74-0.76, \mathrm{n}=8,330$ ).

There has been only one prehospital triage tool for acute disease and trauma in Japan: the prehospital severity and urgency criterion, produced by the Japan Foundation for Ambulance Service Development in 2004 [17]. However, this criterion is complex to use because it is based on 10 categories: trauma, burn, intoxication, consciousness disorder, chest pain, dyspnea, gastrointestinal bleeding, abdominal pain, pregnancy, and infancy; it requires approximately 20 items to be checked. In practice, this cri-

Table 2: Screening parameters according to each cut-off point of prehospital score

\begin{tabular}{ccccc}
\hline Cut off point & \%Sensitivity & \%Specificity & \%PPV & \%NPV \\
\hline$>=1$ & 97.1 & 13.4 & 38.7 & 89.1 \\
$>=2$ & 96.5 & 15.9 & 39.3 & 89.0 \\
$>==3$ & 86.6 & 43.2 & 46.2 & 85.1 \\
$>=4$ & 83.4 & 50.0 & 48.3 & 84.1 \\
$>=5$ & 67.0 & 70.8 & 56.4 & 79.2 \\
$>=6$ & 55.5 & 81.8 & 63.2 & 76.5 \\
$>=7$ & 42.8 & 89.9 & 70.6 & 73.6 \\
$>=8$ & 31.3 & 95.1 & 78.2 & 71.1 \\
$>=9$ & 22.2 & 97.9 & 85.8 & 69.1 \\
$>=10$ & 14.1 & 99.3 & 91.8 & 67.2 \\
$>=11$ & 10.1 & 99.9 & 95.6 & 66.3 \\
$>=12$ & 6.0 & 100 & 98.9 & 65.4 \\
$>=13$ & 5.0 & 100 & 100 & 65.1 \\
$>=14$ & 2.0 & 100 & 100 & 64.4 \\
\hline
\end{tabular}

PPV: Positive predictive value NPV: Negative predictive value $\mathrm{Cl}$ : Confidence interval terion is not used because of its complexity. Moreover, criteria based on chief complaints are of little use for cases with complaints outside those 10 categories, such as paralysis or headache [11].

In contrast, the prehospital score of the present study is a simple to use, comprehensive triage tool for acute disease. Furthermore, emergency personnel without emergency life-saving technician licences could use this scoring system because of its simplicity.

Sensitivity, specificity, PPV and NPV are barometers for the utility of a triage tool. As under-triage is less desirable than over-triage, sensitivity is more important than specificity. When we set the cut-off point at total score $\geq 2$, sensitivity and specificity were respectively $97 \%(2,900 /$ $3,002)$ and $16 \%(841 / 5,328)$. Furthermore, we can also predict the likelihood of death as $0 \%$ and $100 \%$ when the score is low $(\leq 1)$ or high $(\geq 12)$, respectively.

If ambulance transfer is refused for patients with score $\leq 1$, $16 \%(841 / 5,328)$ of cases of inappropriate ambulance usage would be avoided. On the other hand, this decision made $3.5 \%(102 / 3,002)$ into under-triage cases.

Previous studies in western countries have reported prehospital guidelines for predicting the requirement for admission to the emergency department (sensitivity = $90 \%$ and specificity $=37 \%$ ) [11] and a prehospital protocol for predicting the critical event during transport (sensitivity $=95 \%$ and specificity $=33 \%$ ) [12]. These studies relied on detailed protocols according to symptoms. Although simple comparison with our study is difficult because of differences in the medical systems, our scoring system showed higher sensitivity and lower specificity. To avoid under-triage, lower specificity might be inevitable.

However, this study is not without its limitations. First, the scoring system did not include information other than the six fundamental elements. For example, the chief complaint is often strongly associated with outcome: chest pain, paralysis, hematemesis or melena. Patients with gastrointestinal bleeding usually require hospitalization, yet 10 patients in this study with gastrointestinal bleeding scored $\leq 1$. Moreover, 4 cerebral infarction cases, 1 myocardial infarction case, 1 pneumothorax case and 1 tuberculosis case scored $\leq 1$. These cases needed rapid transfer to an emergency hospital. If low-scoring $(<=1)$ patients with chest pain, paralysis, hematemesis or melena were considered as indicating ambulance usage, $17 \%(17 / 102)$ of under-triage cases could be avoided. Since our purpose is to develop a simple triage tool that would be useful for ambulance refusal for Japan emergency personnel regardless of emergency life-saving technician licence, an additional assessment for chief 
complaints would be necessary for practical use. However, sensitivity and specificity did not change substantially when these chief complaints were taken into account (97\% and 15\%, respectively).

Second, the scoring system was mainly based on patient vital signs, but did not include body temperature or respiration rate. The method for measuring body temperature in the prehospital setting is not standardized; some are measured by tympanic temperature, others by axillary temperature. Therefore, we did not use body temperature. The inclusion of respiration rate in the regression model excluded $15 \%$ of the subject data and did not improve the predictive value for hospitalization (sensitivity $=97 \%$ and specificity $=15 \%$ ).

Third, the proportion of emergency hospitalization in Kishiwada City is substantially lower than the national average: $36 \%$ compared with $49 \%$. Criteria for hospitalization vary according to areas and hospitals. Furthermore, we could not obtain complete data for $9 \%$ of patients in Kishiwada City. Therefore, the external validity of the prehospital score is uncertain.

Fourth, we had no follow-up data on patients who were not hospitalized. Some might be misdiagnosed in the emergency room and not hospitalized at that time, but would then be hospitalized after a few days.

Despite these limitations, the present study suggests that a simple score based on 6 fundamental elements enables us to decide whether ambulance transfer is indicated with $97 \%$ sensitivity and 16\% specificity. In the near future, refusal of ambulance transfer for mild patients may be allowed in Japan. Our prehospital score could be a tool for deciding transfer refusal by emergency personnel.

\section{Conclusion}

We have developed a prehospital score, a simple acute disease triage tool for Japan's emergency medical service system. Further research on validity would be necessary.

\section{Competing interests}

The author(s) declare that they have no competing interests.

\section{Authors' contributions}

YM, HT and HF were involved in the acquisition of data. YT was involved in data analysis and drafting the manuscript. TT and HI provided statistical advice on study design and critical revision of the manuscript. All authors read and approved the final manuscript

\section{Acknowledgements}

The authors thank the staff of Kishiwada City Fire Department who recorded the prehospital data. This study was supported by the Grant-in-
Aid for Scientific Research, Ministry of Education, Culture, Sports, Science and Technology, Japan (Grant number: 18659|49).

\section{References}

I. Circumstances of Emergency and Rescue. Agency ofFire and Disaster Management [http://www.fdma.go.jp/neuter/topics/sta tistcs/pdf/hl7 kyukyu kyujo.pdf]. (in Japanese)

2. Lewin MR, Hori S, Aikawa N: Emergency medical services in Japan: an opportunity for the rational development of prehospital care and research. J Emerg Med 2005, 28:237-24I.

3. Pozner CN, Zane R, Nelson S], Levine M: International EMS Systems: The United States: past, present, and future. Resuscitation 2004, 60:239-244.

4. Adnet F, Lapostolle : International EMS Systems: France. Resuscitation 2004, 63:7-9.

5. Black JJM, Davies GD: International EMS Systems: United Kingdom. Resuscitation 2005, 64:21-29.

6. Health and Welfare Statistics Association: Population Statistics. Kosei No Shihyo 2005, 52(9):33-38. (in Japanese)

7. Ohshige K, Mizushima S, Watanabe J, Mukasa M, Kawano T, Sekiguchi $T$, Awashima T, Tochikubo O: Utilization of emergency ambulances in Yokohama city, Japan. Nippon Koshu Eisei Zasshi 200 I, 48:56-64. (in Japanese)

8. Ohsgige K, Tochikubo O: A descriptive study on the trend of ambulance utilization in an aging society, Yokohama, Japan. Yokohama Med Bull 2003, 50: I5-23.

9. Satou O: Consciousness disorder. In Hyojun-Noshinkei-Gekagaku 7th edition. Edited by: Yata K. Tokyo: Igaku-Shoin; 1996:162-176. (in Japanese)

10. Schmidt T, Atcheson R, Federiuk C, Mann NC, Pinney T, Fullaer D, Colbry K: Evaluation of protocols allowing emergency medical technicians to determine need for treatment and transport. Acad Emerg Med 2000, 7:663-669.

II. Pointer JE, Levitt MA, Young JC, Promes SB, Messana BJ, Ader MEJ: Can paramedics using guidelines accurately triage patients? Ann Emerg Med 200I, 38:268-277.

12. Holstein A, Plaschke A, Vogel MY, Egberts EH: Prehospital management of diabetic emergencies-a population-based intervention study. Acta Anaesthesiol Scand 2003, 47:6 I0-6I5.

13. Tirschwell DL, Longstreth WT, Becker KJ, Gammans RE, Sabounjian LA, Hamilton S, Morgenstern LB: Shortening the NIH stroke scale for use in the prehospital setting. Stroke 2002, 33:280I-2806.

14. Llanes JN, Kidwell CS, Starkman S, Leary MC, Eckstein M, Saver JL: The Los Angeles Motor Scale (LAMS): a new measure to characterize stroke severity in the field. Prehosp Emerg Care 2004, 8:46-50.

15. Welsh RC, Ornato J, Armstrong PW: Prehospital management of acute ST-elevation myocardial infarction: A time for reappraisal in North America. American Heart J 2003, I 45: I-8.

16. McVaney KE, Macht M, Clowell CB, Pons PT: Treatment of suspected cardiac ischemia with aspirin by paramedics in an urban emergency medical services system. Prehosp Emerg Care 2005, 9:282-284.

17. The prehospital severity and urgency criteria. Foundation of Ambulance Service Development [http://www.fasd.or.jp/
A tyousa/hanso0l.pdf]. (in Japanese)

\section{Pre-publication history}

The pre-publication history for this paper can be accessed here:

http://www.biomedcentral.com/1471-227X/7/17/pre pub 